\title{
Práticas alimentares nos dois primeiros anos de vida: presença de vulnerabilidade em saúde
}

\author{
Food practices in the first two years of life: presence of vulnerability in health
}

Roberta Lamonatto Taglietti ${ }^{1}$, Karine Lazarotto ${ }^{2}$, Silvia Letícia Bernardi Pinto ${ }^{2}$, Carla Rosane Paz Arruda Teo ${ }^{3}$

${ }^{1}$ Mestranda do Programa de Pós-Graduação em Ciências da Saúde da Universidade Comunitária da Região de Chapecó (UNOCHAPECÓ), Chapecó, Santa Catarina; ${ }^{2}$ Acadêmicas do curso de Nutrição da UNOCHAPECÓ, Chapecó, Santa Catarina; ${ }^{3}$ Docente do Programa de Pós-Graduação em Ciências da Saúde da UNOCHAPECÓ, Chapecó, Santa Catarina.

\section{RESUMO}

Objetivos: Investigar as práticas alimentares nos dois primeiros anos de vida em crianças atendidas por um serviço público de saúde.

Métodos: Este foi um estudo transversal, realizado a partir de entrevistas com mães de crianças com idade de seis a 24 meses incompletos, recrutadas entre os meses de fevereiro e junho de 2013, na Unidade Básica de Vigilância em Saúde do município de São Miguel do Oeste, estado de Santa Catarina. Como instrumento de coleta utilizou-se um questionário de frequência do consumo alimentar do Sistema de Vigilância Alimentar e Nutricional (SISVAN) aplicado às mães das crianças.

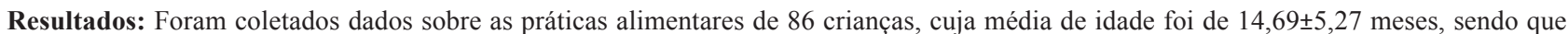
52 crianças $(60,46 \%)$ tinham idade inferior a 12 meses. A maioria seguia boas práticas alimentares, como ingestão de frutas $(91,86 \%)$, carne $(84,88 \%)$ e verduras/legumes $(82,55 \%)$. Entretanto, foi encontrada uma proporção relevante de práticas inadequadas, como ver televisão durante as refeições $(33,72 \%)$, ingestão de sucos industrializados $(47,67 \%)$, ingestão de refrigerantes $(46,51 \%)$ e menos de seis meses de aleitamento materno exclusivo $(56,70 \%)$.

Conclusões: Embora algumas práticas alimentares saudáveis tenham sido prevalentes no grupo avaliado, foi encontrada uma proporção relevante de práticas pouco saudáveis. Este é um achado preocupante em crianças menores de dois anos, dada a vulnerabilidade dessa fase da vida e sua importância para a construção de padrões alimentares que têm grande possibilidade de se perpetuarem ao longo da vida do indivíduo.

DESCRITORES: NUTRIÇÃO INFANTIL; HÁBITOS ALIMENTARES; VULNERABILIDADE EM SAÚDE.

\section{ABSTRACT}

Aims: To investigate feeding practices in the first two years of life in children treated by a public health service.

Methods: This was across-sectional study based on interviews with mothers of children aged six to incomplete 24 months, recruited between February and June 2013, in the Basic Health Surveillance Unit of the city of Sao Miguel do Oeste, Santa Catarina state. A food frequency intake questionnaire of the Food and Nutrition Surveillance System (SISVAN - Sistema de Vigilância Alimentar e Nutricional), applied to the mothers, was used as the data collection instrument.

Results: Data on feeding practices were collected from 86 children, whose average age was $14.69 \pm 5.27$ months, and 52 children (60.46\%) were younger than 12 months. The majority had good dietary practices, such as intake of fruits $(91.86 \%)$, meat (84.88\%) and vegetables/ salads $(82.55 \%)$. However, a significant proportion of inappropriate practices was found, such as watching television during meals (33.72\%), ingestion of processed juices (47.67\%), soft drink intake (46.51\%) and less than six months exclusive breastfeeding (56.70\%).

Conclusions: Although some healthy dietary practices have been prevalent in the group evaluated, a significant proportion of unhealthy eating practices was found. This is a concerning finding in children under two years, given the vulnerability of this phase of life and its importance to the construction of dietary patterns that have great possibility to perpetuate over the life of the individual.

KEY WORDS: INFANT NUTRITION; FOOD HABITS; HEALTH VULNERABILITY. 


\section{INTRODUÇÃO}

Atualmente, a manutenção do aleitamento materno exclusivo é recomendada até o sexto mês de vida da criança. Após esse período e até os dois anos de idade está indicado o aleitamento materno complementado, fase em que os alimentos oferecidos à criança devem complementar o aporte nutricional representado pelo leite materno. Neste sentido, a alimentação complementar é fundamental para promover o crescimento e o desenvolvimento. A alimentação complementar aproxima a criança dos hábitos alimentares de seus cuidadores; assim, orientar e promover a adesão dos responsáveis pela criança, garantindo uma adequada introdução de alimentos nessa fase da vida, constitui-se em um desafio para os profissionais de saúde. ${ }^{1}$

É importante destacar que a alimentação associa-se ao perfil de saúde e nutrição, especialmente de crianças menores de dois anos de idade, entre as quais as práticas alimentares inadequadas estão relacionadas ao aumento da morbidade. ${ }^{2,3}$ Segundo Bercini et al., ${ }^{3}$ a formação do hábito alimentar da criança é feita por meio da interação da mesma com o alimento, pelo seu desenvolvimento anatomofisiológico e por fatores emocionais, psicológicos, socioeconômicos e culturais. $\mathrm{O}$ mais relevante de todos é a influência da própria mãe e de pessoas ligadas à sua alimentação, sendo a família responsável pela transmissão da cultura alimentar à criança. ${ }^{4-6}$ Crianças cujas mães têm baixa escolaridade apresentam maior consumo de guloseimas entre refeições, enquanto mães com maior escolaridade costumam ser mais restritivas quanto ao consumo de açúcar na infância, o que evidencia o importante papel da mãe na construção de práticas alimentares infantis mais saudáveis. ${ }^{7,8}$

Nos últimos anos têm ocorrido avanços importantes na promoção da amamentação, mas as intervenções visando promover práticas saudáveis de alimentação na primeira infância não têm recebido atenção suficiente e nem sempre são efetivas, sendo elevada a frequência de inadequações quanto às práticas de alimentação complementar, as quais constituem fatores de vulnerabilidade para a saúde infantil, com prováveis repercussões ao longo da vida. ${ }^{9,10}$

O marco teórico da vulnerabilidade considera a chance das pessoas adoecerem, sendo resultante de um conjunto de aspectos individuais, coletivos e contextuais que acarretam maior ou menor suscetibilidade ao adoecimento, assim como maior ou menor disponibilidade de recursos de proteção. Considerando as dimensões da vulnerabilidade no que diz respeito à alimentação da criança, destaca-se o componente individual, que se refere à capacidade da família acessar e elaborar infor- mações, incorporando-as ao cotidiano do cuidado à criança, o que depende, entre outros aspectos, do nível de escolaridade, dos valores e das crenças da família. ${ }^{8,11-13}$

Em relação ao componente social da vulnerabilidade, destacam-se as referências culturais da família, o acesso a emprego, educação, o suporte social disponível, entre outros fatores. Já quanto ao componente programático, salientam-se o acesso a serviços de saúde, a qualidade destes serviços e as políticas e programas de saúde específicos. ${ }^{14,}{ }^{15}$ No âmbito deste marco teórico, e retomando a questão da alimentação infantil, pontua-se que, do ponto de vista nutricional, a introdução precoce de alimentos pode colocar a criança em estado vulnerável por diminuir a duração do aleitamento materno, interferir na absorção de nutrientes e, ainda, aumentar o risco de contaminação e de reações alérgicas. Por outro lado, a introdução tardia de alimentos também é desfavorável e vulnerabiliza a criança, na medida em que não são atendidas as suas necessidades nutricionais. ${ }^{16}$

Este estudo teve como objetivo investigar as práticas alimentares de crianças de seis a 24 meses de idade atendidas por um serviço público de saúde de um município da região Extremo Oeste do estado de Santa Catarina, de forma a identificar em que medida essas práticas indicam situações de vulnerabilidade em saúde para esse grupo.

\section{MÉTODOS}

Este foi um estudo transversal, realizado em São Miguel do Oeste, um município da região Extremo Oeste de Santa Catarina, a partir de entrevistas com mães de crianças com idade de seis a 24 meses incompletos. A escolha recaiu sobre esta faixa etária por ser o período de introdução de alimentos complementares e, portanto, uma fase crucial para a formação do hábito alimentar da criança. De acordo com dados obtidos do Sistema de Informação da Atenção Básica do Ministério da Saúde (SIAB), o total de crianças nessa faixa etária no município era de 412 crianças à época da coleta dos dados de pesquisa. ${ }^{17}$

O censo de 2010 mostra uma população de 35 mil habitantes $(51,4 \% \text { de mulheres e } 48,6 \% \text { de homens })^{18}$ e um taxa de urbanização de $79,6 \%$ para o município de São Miguel do Oeste. O Índice de Desenvolvimento Humano Municipal é considerado muito alto, de 0,801 , tendo evoluído $42,0 \%$ nas últimas duas décadas, o que indica avanços no desenvolvimento social e econômico da população, advindos principalmente de incrementos nos componentes educação $(103,0 \%)$ e renda $(26,1 \%)$ nesse período. ${ }^{19}$ 
Os dados foram coletados entre os meses de fevereiro e junho de 2013, na Unidade Básica de Vigilância em Saúde onde está localizada a central de vacinas, uma vez que esta é uma unidade central, que abrange a maior população do município. Além desse local, dados foram coletados também na Unidade da Pastoral da Criança do município, de maneira a atingir um maior número de mães e crianças. A população que participou do estudo é a que procurou algum tipo de atendimento na Unidade de Saúde ou na Pastoral da Criança durante o período de pesquisa. A coleta foi realizada em cinco inserções das pesquisadoras nos espaços acima citados, sendo uma delas no dia do encontro da Pastoral da Criança ocorrido nos meses da coleta, uma no dia da Campanha Nacional de Vacinação e as demais ocorreram em três dias aleatórios durante o período de pesquisa.

O projeto que originou este estudo foi aprovado por Comitê de Ética em Pesquisa da Universidade Comunitária da Região de Chapecó (UNOCHAPECÓ), sob o parecer $n^{\circ} 319 / 2012$. Ao procurarem os locais de coleta de dados, as mães que traziam crianças na faixa etária entre seis meses e dois anos eram convidadas a participar do estudo pelas pesquisadoras, que esclareciam os objetivos e procedimentos da pesquisa. As mães que aceitaram assinaram um termo de consentimento livre e esclarecido, e seus filhos compuseram a amostra do estudo. Os procedimentos empregados, tanto os de abordagem das mães quanto os de coleta de dados, foram previamente autorizados pela prefeitura do município.

Para a coleta de dados foi empregado o questionário do Sistema de Vigilância Alimentar e Nutricional (SISVAN) para crianças de seis a 24 meses. O instrumento contém seis questões que se referem às práticas alimentares do dia anterior, assumindo o pressuposto de que este foi um dia típico no cotidiano alimentar da criança e, portanto, representa sua alimentação habitual. Além destas, o questionário contém duas perguntas que se referem à introdução de alimentação complementar antes dos seis meses, e duas que investigam o consumo mensal de alimentos industrializados. ${ }^{20}$

Os dados obtidos foram tabulados em planilha do Programa Microsoft Excel ${ }^{\circledR}$, analisados por meio de estatística descritiva (frequências absoluta e relativa) e comparados com as recomendações do "Guia Alimentar para crianças menores de dois anos" do Ministério da Saúde. ${ }^{17}$

\section{RESULTADOS}

O total de entrevistas realizadas foi de 86 , compondo-se um grupo que correspondeu a $20,87 \%$ da população estimada para a faixa etária (de seis meses a dois anos) no município. A média de idade das crianças participantes foi de 14,69 $\pm 5,27$ meses, sendo que 52 crianças $(60,46 \%)$ tinham idade inferior a 12 meses. Das mães entrevistadas, $60(69,76 \%)$ referiram trabalhar fora.

A expressiva maioria das mães entrevistadas relatou que seus filhos haviam consumido verduras/legumes, frutas, carne e feijão no dia anterior, alimentos assumidos como marcadores de alimentação saudável. Considerando-se a faixa etária alvo deste estudo, apenas o consumo de leite de vaca no dia anterior ao da entrevista configurou-se como prática alimentar inadequada prevalente no grupo avaliado. As outras práticas pouco saudáveis aparecem com frequências não prevalentes, embora relevantes, e incluem mães que relataram que seus filhos foram alimentados no dia anterior enquanto assistiam à televisão, assim como aquelas que informaram o consumo de sucos industrializados e refrigerantes no último mês. Além destas práticas, chamam a atenção, ainda, no que diz respeito à introdução de alimentação complementar, os percentuais de crianças que receberam alimentos açucarados e ou comida salgada precocemente, antes dos seis meses (Tabela 1).

Tabela 1. Práticas alimentares de 86 crianças de 6 a 24 meses,atendidas na rede pública de saúde de São Miguel do Oeste, município da região Extremo Oeste de Santa Catarina. Fevereiro a junho de 2013.

\begin{tabular}{|lcccc}
\multicolumn{1}{c}{ Perguntas } & \multicolumn{3}{c}{ Sim } & \multicolumn{3}{c}{ Não } \\
\cline { 2 - 5 } & $\mathbf{n}$ & $\%$ & $\mathbf{n}$ & \% \\
\hline A criança tomou leite de vaca ontem? & 77 & 89,53 & 9 & 10,47 \\
\hline A criança comeu verduras/ legumes ontem? & 71 & 82,55 & 15 & 17,44 \\
\hline A criança comeu frutas ontem? & 79 & 91,86 & 7 & 8,13 \\
\hline A criança comeu carne ontem? (boi, porco, frango, peixe, miúdos, outros) & 73 & 84,88 & 13 & 15,11 \\
\hline A criança comeu feijão ontem? & 81 & 94,18 & 5 & 5,81 \\
\hline A criança comeu assistindo televisão ontem? & 29 & 33,72 & 57 & 66,28 \\
\hline A criança recebeu alimentos açucarados antes dos 6 meses? & 30 & 34,88 & 56 & 65,12 \\
\hline A criança recebeu comida salgada antes dos 6 meses? & 40 & 46,51 & 46 & 53,49 \\
\hline A criança tomou suco industrializado no último mês? & 41 & 47,67 & 45 & 52,33 \\
\hline A criança tomou refrigerante no último mês? & 35 & 40,69 & 51 & 59,30 \\
\hline
\end{tabular}


Em relação à duração do aleitamento materno exclusivo, 48 mães $(56 \%)$ relataram suspensão do aleitamento materno exclusivo antes dos seis meses completos, sendo que 17 mães $(19,7 \%)$ referiram que este cessou antes dos quatro meses. Vinte e oito crianças $(32,55 \%)$ receberam aleitamento materno exclusivo até os seis meses, enquanto em 10 crianças $(11,62 \%)$ a alimentação com leite materno sem a introdução de alimentos complementares foi mantida além dos seis meses (Figura 1).

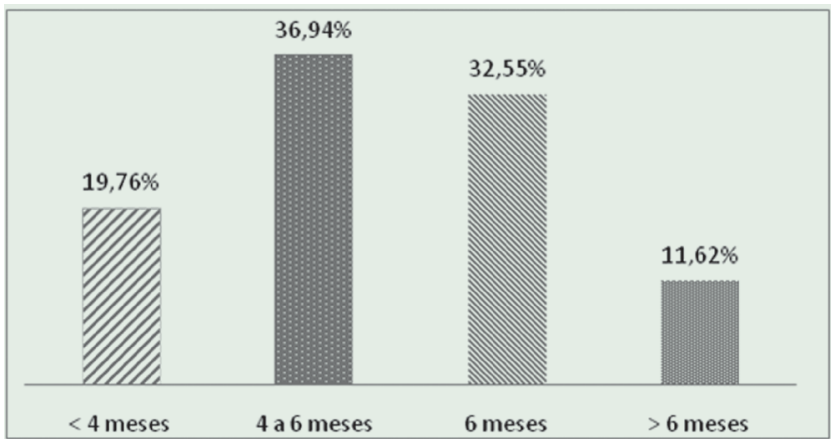

Figura 1. Período de aleitamento materno exclusivo entre 86 crianças de 6 a 24 meses, atendidas entre fevereiro e junho de 2013 na rede pública de saúde de São Miguel do Oeste, município da região Extremo Oeste de Santa Catarina.

\section{DISCUSSÃO}

De acordo com o guia alimentar para crianças menores de dois anos do Ministério da Saúde, somente a partir dos seis meses o organismo da criança está preparado para receber alimentos diferentes do leite materno, chamados alimentos complementares, que têm a finalidade de complementar o leite materno e não de substituí-lo. ${ }^{21}$ Os dados do presente estudo indicam baixa adesão ao aleitamento materno exclusivo pelo menos até o sexto mês, o que configura prática alimentar de risco.

Em estudo realizado por Garcia et al., ${ }^{22}$ o início do aleitamento materno ao nascer foi referido por $99,0 \%$ das mães; já a prática do aleitamento materno exclusivo até o sexto mês foi relatada por apenas 9,64\% delas. ${ }^{22}$ Segundo a II Pesquisa de Prevalência de Aleitamento Materno nas Capitais Brasileiras e Distrito Federal, ${ }^{23}$ a duração mediana do aleitamento materno exclusivo foi de 1,8 meses e a duração mediana do aleitamento materno de 11,2 meses. A prevalência do aleitamento materno exclusivo em menores de seis meses foi de $41 \%$ no conjunto das capitais brasileiras e de $52,4 \%$ em Florianópolis. ${ }^{23} \mathrm{~A}$ interrupção do aleitamento materno exclusivo vem sendo um dos mais importantes problemas de saúde pública no país, ${ }^{24}$ acompanhado da introdução precoce de alimentos, privando a criança de receber, em maior quantidade, o leite materno..$^{25}$

Nos últimos anos, vários estudos vêm demonstrando a ocorrência de agravos significativos quando a amamentação não acontece, considerando entre muitos fatores as suas características nutricionais ideais para a criança, sendo que o principal argumento contra a introdução dos alimentos complementares antes do sexto mês de vida é o aumento da morbidade e mortalidade infantil. ${ }^{3,26}$

Destaca-se ainda, neste estudo, que 40 bebês (46,51\%) iniciaram a alimentação complementar com alimentos salgados antes dos seis meses de idade, situação esta que pode interferir na absorção de nutrientes, aumento do risco de alergia alimentar, e maior ocorrência de doenças crônico-degenerativas na idade adulta. ${ }^{27}$ Esses achados apontam para a condição de vulnerabilidade do grupo avaliado, com destaque para a introdução precoce de alimentação complementar, resultando em expressiva proporção de crianças que recebeu aleitamento materno exclusivo por menos de seis meses e, inclusive, por menos de quatro meses. Entretanto, também é preocupante a constatação de que $11,62 \%$ das crianças continuaram a receber aleitamento materno exclusivo além dos seis meses, o que também não é adequado, uma vez que após essa idade, o leite materno passa a não atender às necessidades nutricionais da criança. ${ }^{1,16}$

Estudo realizado por Corrêa et al. ${ }^{28}$ também encontrou dados significativos, demonstrando que $80 \%$ das 516 crianças pesquisadas receberam fruta e $77,5 \%$ receberam suco natural de forma associada ao aleitamento materno antes de completarem seis meses. No mesmo estudo, os autores reportaram que $36,8 \%$ das crianças receberam leite modificado em substituição ao aleitamento materno exclusivo, tendo sido as sopas introduzidas entre os 6,2 e os 6,8 meses.

No presente estudo, o leite de vaca faz parte da alimentação de $89,53 \%$ das crianças pesquisadas, sendo que seu consumo é contraindicado até os 12 meses de vida. Este achado é impactante quando se observa que mais da metade das crianças participantes da pesquisa tinham idade inferior a 12 meses. $O$ fato ilustra a relativa inadequação das escolhas alimentares para crianças desta idade, uma vez que o leite de vaca vem sendo associado a altos índices de anemia na infância. Em estudo realizado por Moy, ${ }^{29} \mathrm{chamou}$ atenção o elevado percentual de crianças que recebiam leite de vaca integral no primeiro ano de vida, com ou sem adição de carboidratos simples, sendo esse um 
fator de risco independente para o desenvolvimento de anemia carencial ferropriva.

Outro complicador para o consumo de leite de vaca nesta fase da vida é apresentado pelo estudo de Saldiva et al. ${ }^{16}$ o qual refere que, aos seis meses de idade, a probabilidade de a criança consumir refeições baseadas somente em leites e mingaus é de $82 \%$, indicando a possibilidade de não se alcançar o aporte necessário de nutrientes em uma fase de acelerados crescimento e desenvolvimento. ${ }^{16}$

O guia alimentar para crianças menores de dois anos recomenda ainda que as crianças devem se acostumar a comer frutas, verduras e legumes desde cedo, pois esses alimentos são importantes fontes de vitaminas, cálcio, ferro, e fibras, ${ }^{21}$ sendo que o consumo inadequado de frutas e hortaliças está entre os 10 principais fatores de risco para a carga total global de doença em todo o mundo. ${ }^{30}$ Cabe destacar que, no presente estudo, $8,13 \%$ das crianças não receberam frutas e $17,44 \%$ delas não receberam verduras e legumes no dia anterior à entrevista. Estas proporções, apesar de serem relativamente baixas na comparação com a literatura sobre o tema, são indicativas de que estes grupos alimentares não fazem parte habitualmente do consumo diário de algumas crianças em uma fase crucial para o seu crescimento e desenvolvimento saudáveis. Recomenda-se, inclusive, que o consumo de frutas, verduras e legumes seja diário. $^{21}$

Estudo realizado por Antunes et al. ${ }^{31}$ revelou que as crianças não atingiram as recomendações de consumo para hortaliças do guia nacional e apresentaram consumo elevado de alimentos de alta densidade calórica, ricos em gorduras e açúcares, a exemplo da população brasileira, o que também tem sido apontado pelas sucessivas Pesquisas de Orçamentos Familiares realizadas no país. ${ }^{32}$

Os resultados do presente estudo parecem refletir essa transformação, uma vez que mais de um terço das crianças receberam alimentos açucarados antes dos seis meses de idade, e quase a metade consumiram sucos industrializados e refrigerantes no último mês. Com relação aos alimentos açucarados, Toloni et al. ${ }^{33}$ referem que o uso precoce de açúcar, principalmente no chá e no leite não materno, faz parte de hábitos culturalmente estabelecidos e da tendência das mães de oferecerem alimentos doces, para, na sua concepção, satisfazer o paladar da criança e deixá-la bem alimentada. No que tange ao consumo de sucos industrializados e refrigerantes, as frequências observadas nesta pesquisa são semelhantes aos achados reportados por Toloni et al. ${ }^{33}$
Adicionalmente, Tuma et al. ${ }^{34}$ observaram, em estudo realizado em Brasília com crianças menores de dois anos, a introdução precoce de alimentos como fastfood, refrigerantes, enlatados e doces ou guloseimas. Os padrões inadequados de consumo têm sido considerados os principais fatores responsáveis pelo aumento da prevalência de obesidade infantil. ${ }^{35}$ Estudo de Bortolini et al., ${ }^{36}$ que objetivou avaliar o consumo alimentar em crianças brasileiras de seis a 59 meses de idade, por região e zona de residência, identificou que aproximadamente $50 \%$ das crianças de todas as regiões do Brasil, com exceção da região Norte, consomem biscoitos ou bolachas diariamente, e $36,4 \%$ das crianças da região Sul consomem diariamente refrigerantes. ${ }^{36}$

O consumo de alimentos industrializados, sejam estes ricos em açúcares simples, gorduras ou aditivos alimentares, representa um conjunto de fatores agressores à saúde da criança, podendo ser a causa do surgimento, ainda na infância ou na vida adulta, de doenças crônicas não transmissíveis, além de conduzir a criança à consolidação de um padrão alimentar pouco saudável que poderá se perpetuar pelos demais ciclos de sua vida.

No desenvolvimento desta pesquisa, foi possível identificar como uma questão social com potencial impacto na alimentação da criança o fato de praticamente $70 \%$ das mães trabalharem fora do lar. Além das prováveis repercussões na formação do hábito alimentar da criança, esta condição tem sido reconhecida como uma importante causa de desmame precoce, anterior aos quatro meses de vida. Estudo realizado por Corrêa et al. ${ }^{28}$ sinalizou que as mães que exerciam atividade fora do lar apresentavam mais chances de oferecer alimentos dos grupos de gorduras, doces, lácteos, amidos, panificados, vegetais e frutas, sendo que leite não materno e frutas foram oferecidos antes dos seis meses, interrompendo o aleitamento materno exclusivo, e os outros alimentos antes dos sete meses.

Outro ponto importante de discussão quanto à formação de hábito alimentar saudável é o registro, nesta pesquisa, de que $33,52 \%$ das crianças se alimentavam assistindo televisão, o que é considerado um comportamento de risco. Mondini et al. ${ }^{37}$ apontaram como um dos principais fatores independentes associados ao risco de sobrepeso das crianças o fato de assistir televisão por mais de quatro horas diárias. Reconhece-se que é provável que essa associação seja devida, em boa parte, à publicidade relativa a alimentos de baixo valor nutricional. Pesquisa de Motta et al. ${ }^{38}$ mostrou que, na percepção dos respon- 
sáveis pelas crianças, a televisão influencia nos hábitos de consumo alimentar no cotidiano das crianças brasileiras. Já está bem demonstrado que quanto maior o tempo dedicado em frente à televisão, menor é o consumo de frutas e vegetais por crianças e adolescentes. Além disso, o consumo de doces, salgadinhos, refrigerantes e alimentos gordurosos é maior na medida em que aumenta o tempo despendido com a televisão. ${ }^{39}$

Concluindo, observou-se neste estudo que algumas práticas alimentares adequadas foram prevalentes. Contudo, considera-se relevante a frequência obser- vada de práticas pouco saudáveis, na medida em que se referem a crianças menores de dois anos, dada a vulnerabilidade inerente a essa faixa etária e sua importância para a construção de padrões alimentares que têm grande possibilidade de se perpetuarem ao longo da vida. As inadequações alimentares constatadas indicam a existência de fatores de vulnerabilidade situados na dimensão individual, a qual diz respeito à capacidade das famílias de aplicarem na vida cotidiana informações adequadas e suficientes para assegurarem uma alimentação saudável às suas crianças, reduzindo suas vulnerabilidades em saúde.

\section{REFERÊNCIAS}

1. Brasil. Ministério da Saúde. Secretaria de atenção à saúde. Departamento de atenção básica. Saúde da criança: nutrição infantil: aleitamento materno e alimentação complementar [Internet]. Brasília; 2009. [acessado 25 de junho de 2013]. Disponível em: http://bvsms.saude.gov. br/bvs/publicacoes/saude_crianca_nutricao_aleitamento_alimentacao.pdf

2. Brasil. Ministério da Saúde. Estratégia nacional para alimentação complementar saudável (ENPACS) Caderno do tutor [Internet]. Brasília, 2010. [acessado 25 de junho de 2013]. Disponível em: http://189.28.128.100/nutricao/docs/geral/caderno_do_tutor.pdf

3. Lamberti LM, Fischer Walker CL, Noiman A, Victora C, Black RE. Breastfeeding and the risk for diarrhea morbidity and mortality. BMC Public Health. 2011;11 Suppl 3:S15. doi: 10.1186/1471-2458-11-S3-S15.

4. Bercini LO, Masukawa TML, Martins MR, Labegalini MPC, Alves NB. Alimentação da criança no primeiro ano de vida em Maringá, PR. Cienc Cuid Saude. 2007;6(2):404-10.

5. Ramos M, Stein L. Desenvolvimento do comportamento alimentar infantil. J Pediatr. 2000;73(3):229-37.

6. Horta BL, Victora CG, Gigante DP, Santos J, Barros FC. Duração da amamentação em duas gerações. Rev Saude Publica. 2007;41(1):13-8.

7. Feldens CA, Hommerding LPB, Santos BZ, Feldens EG,Vítolo MR. Práticas alimentares cariogênicas e fatores associados em crianças do Sul do Brasil. Pesq Bras Odonto Ped Clin Integr. 2010;10(2):201-7.

8. Romulus-Nieuwelink JJ, Doak C, Albernaz E, Victora CG, Haisma H. Breast milk and complementary food intake in Brazilian infants according to socio-economic position. Int J Pediatr Obes. 2011;6(2-2):e508-14.

9. Monte C,Guiugliani E. Recomendações para alimentação complementar da criança em aleitamento materno. J Pediatr (Rio J). 2005;80(5):131-41.

10. Euclydes, MP. Nutrição do lactente - base científica para uma alimentação adequada. $3^{\text {a }}$ ed. Viçosa: Editora UFV; 2005.

11. Ayres JRCM, França JI, Calazans GJ, Saletti FHC. O conceito de vulnerabilidade e as práticas de saúde: novas perspectivas e desafios. In: Czeresnia D, Freitas C, organizadores. Promoção da Saúde: conceitos, reflexões e tendências. Rio de Janeiro: Fiocruz; 2003. p. 117-39.

12. Motta MGC, Diefenbach GDF. Dimensões da vulnerabilidade para as famílias da criança com dor oncológica em ambiente hospitalar. Esc Anna Nery. 2013;17(3):482-90.

13. Paiva V, Ayres J R, Buchalla CM. Vulnerabilidade e direitos humanos - prevenção e promoção da saúde da doença à cidadania. Curitiba: Juruá; 2012.

14. Ayres JRCM, Calzans GJ, Saletti HCF, França IJ. Risco, vulnerabilidade e práticas de prevenção e promoção da saúde. In: CAMPOS, G.W.S (Org.) Tratado de saúde coletiva. $2^{\text {a }}$ ed. São Paulo/Rio de Janeiro: Hucitec; 2009. p.375-417.

15. Albernaz E, Araújo CL, Tomasi E, Mintem G, Giugliani E, Matijasevich A, Onis Md, Barros FC, Victora CG. Influence of breastfeeding support on the tendencies of breastfeeding rates in the city of Pelotas (RS), Brazil, from 1982 to 2004. J Pediatr (Rio J). 2008;84(6): $560-4$

16. Saldiva SR, Escuder MM, Mondini L, Levy RB, Venancio SI. Práticas alimentares de crianças de 6 a 12 meses e fatores maternos associados. J Pediatr. 2007;83(1):53-8.

17. Brasil. Ministério da Saúde. Sistema de informação da atenção básica - SIAB [Internet]. Santa Catarina; 2013 [Citado20 de fevereiro de 2013]. Disponível em: http://tabnet.datasus.gov.br/cgi/deftohtm.exe?siab/cnv/SIABSSC.def

18. Brasil. Instituto Brasileiro de Geografia e Estatística-IBGE. Contagem da população no censo [Internet]. Santa Catarina; 2010. [Citado 31 de julho de 2013]. Disponível em:http://www.censo2010.ibge.gov.br/sinopse/index.php?dados=26\&uf=42\#topo_piramide

19. Brasil. Programa das Nações Unidas para o Desenvolvimento-PNUD. Atlas de Desenvolvimento Humano [Internet]. São Paulo; 2013. [acessado 31 de julho de 2013]. Disponível em: http://www.pnud.org.br/IDH/Atlas2003.aspx?indiceAccordion=1\&li=li_Atlas>

20. Brasil. Ministério da Saúde. Secretaria de atenção à saúde. Protocolos do sistema de vigilância alimentar e nutricional-SISVAN [Internet]. Brasília; 2008. [acessado 10 de março de 2013]. Disponível em: http://bvsms.saude.gov.br/bvs/publicacoes/protocolo_sistema_vigilancia_ alimnetar.pdf 
21. Brasil. Ministério da Saúde. Dez passos para uma alimentação saudável: guia alimentar para menores de 2 anos [Internet]. Brasília; 2013. [acessado 20 de março de 2013]. Disponível em: http://bvsms.saude.gov.br/bvs/publicacoes/dez_passos_alimentacao_saudavel_guia.pdf

22. Garcia MT, Granado FS, Cardoso MA. Alimentação complementar e estado nutricional de crianças menores de dois anos atendidas no programa saúde da família em Acrelândia, Acre, Amazônia Ocidental Brasileira. Cad Saúde Pública. 2011;27(2):305-16.

23. Brasil. Ministério da Saúde. II Pesquisa de Prevalência de Aleitamento Materno nas Capitais Brasileiras e Distrito Federal. Brasília, 2009. Disponível em http://bvsms.saude.gov.br/bvs/publicacoes/pesquisa_prevalencia_aleitamento_materno.pdf

24. Parizoto G, Parada C, Venâncio S, Carvalhaes MA. Tendência e determinantes do aleitamento materno exclusivo em crianças menores de 6 meses. J Pediatr (Rio J). 2009;55(3):201-8.

25. Ferreira JV, Castro LMC, Menezes MFG. Alimentação no primeiro ano de vida: a conduta dos profissionais de saúde e a prática exercida pela família. Ceres. 2009;4(3):117-29.

26. Dias MCAP, Freire LMS, Franceschini SCC. Recomendações para alimentação complementar de crianças menores de dois anos. Rev Nutr. 2010;23(3):475-86.

27. Vieira RW, Dias RP, Coelho SC, Ribeiro RL. Do aleitamento materno à alimentação complementar: atuação do profissional nutricionista. Rev Saúde Amb. 2009;4(2):1-8.

28. Corrêa EM, Corso ACMT, Moreira EAM, Kazap IAM. Alimentação complementar e características maternidade crianças menores de dois anos de idade em Florianópolis (SC). Rev Paul Pediatr. 2009;27(3):258-64.

29. Moy DJ. Prevalence, consequences and prevention of childhood nutrition iron deficiency: a child public health perspective. Clin Lab Haematol. 2006;28(5):291-8.

30. Jaime PC, Figueiredo ICR, Moura EC, Malta DC. Fatores associados ao consumo de frutas e hortaliças no Brasil, 2006. Rev Saúde Pública. 2009;43(12):57-64.

31. Antunes ML, Salles CR, Mello MA, Sichieri R. Comparação de dois programas computacionais utilizados na estimativa do consumo alimentar de crianças. Rev Bras Epidemiol. 2007;10(2):267-75.

32. Levy RB, Moreira CR, Mondini L, Sichieri R, Monteiro CA. Distribuição regional e socioeconômica da disponibilidade domiciliar de alimentos no Brasil em 2008-2009. Rev Saúde Pública. 2012;4(1):6-15.

33. Toloni MHA, Longo SG, Goulart RMM, Taddei JAAC. Introdução de alimentos industrializados e de alimentos de uso tradicional na dieta de crianças de creches públicas no município de São Paulo. Rev Nutr. 2011;24(1):61-70.

34. Tuma RCFB, Costa THM, Schmitz BAS. Avaliação antropométrica e dietética de pré-escolares em três creches de Brasília, Distrito Federal. Rev Bras Saude Mater Infant. 2005;5(4):419-28.

35. Santos JV, Gigante DP, Domingues MR. Prevalência de segurança alimentar em Pelotas, Rio Grande do Sul, Brasil, e estado nutricional de indivíduos que vivem nessa condição. Cad Saúde Pública. 2010;26(1):41-49.

36. Bortolini GA, Gubert MB, Santos LMP. Consumo alimentar entre crianças brasileiras com idade de 6 a 59 meses. Cad Saúde Pública. 2012;28(9):1759-71.

37. Mondini L, Levy RB, Saldiva SRDM, Venâncio SI, Aguiar JA, Stefanini MLR. Prevalência de sobrepeso e fatores associados em crianças ingressantes no ensino fundamental em um município da região metropolitana de São Paulo, Brasil. Cad Saúde Pública. 2007;23(8):1825-34

38. Motta GS, Gallo P, Cuenca A. Influência da televisão nos hábitos alimentares de crianças do nordeste brasileiro. Rev Bras Crescim Desenvolv Hum. 2013;23(1):87-93.

39. Rossi CE, Albernaz DO, Vasconcelos FAG, Assis MAA, Pietro PFD. Influência da televisão no consumo alimentar e na obesidade em crianças e adolescentes: uma revisão sistemática. Rev Nutr. 2010;23(4):607-20. 